\title{
Multicenter Hemophilia Cohort Study
}

National Cancer Institute

\section{Source}

National Cancer Institute. Multicenter Hemophilia Cohort Study. NCI Thesaurus. Code C15814.

A multi-center study of HIV disease progression and heterosexual transmission begun in 1982. 